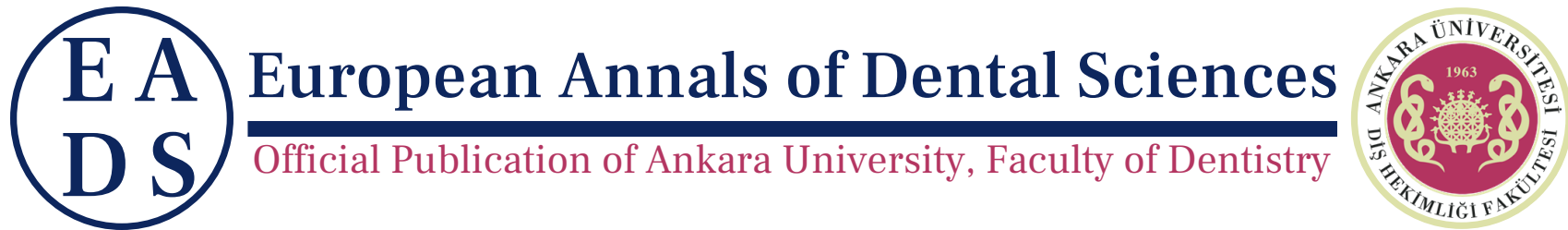

EADS, 2021, 48 (3), 101-107

\title{
Comparison of the Effectiveness of Chemo-mechanical and Traditional Caries Removal Methods in Primary Teeth Using Micro-Computed Tomography
}

\author{
Melike Turgut Coşgun ${ }^{1}$,* , Firdevs Tulga Öz ${ }^{1}$, Mert Ocak ${ }^{2}$ and Kaan \\ Orhan $\odot 3,4$
}

${ }^{1}$ Department of Pedodontics, Faculty of Dentistry, Ankara University, Ankara, Turkey and ${ }^{2}$ Department of Anatomy, Faculty of Dentistry, Ankara University, Ankara, Turkey and ${ }^{3}$ Department of Dentomaxillofacial Radiology, Faculty of Dentistry, Ankara University, Ankara, Turkey and ${ }^{4}$ Ankara University Medical Design Application and Research Center (MEDITAM), Ankara, Turkey

*Corresponding Author; mlktrgt@gmail.com

\begin{abstract}
Purpose: The purpose of this study was to evaluate dentin mineral density before and after caries removal with the traditional caries removal technique and chemo-mechanical method, with or without the use of caries detection dye. Our null hypothesis was that the chemo-mechanical method with minimally invasive treatment would achieve an effect similar to that of conservative pediatric dentistry treatment.

Materials \& Methods: Forty-eight medium-sized, interfacial decayed primary molar teeth without pulpal perforation were selected. Two groups were formed by Papacarie Duo and conventional method; then four sub-groups were formed by two caries detection dye applied or not applied groups, twelve teeth were randomly divided into each group. The teeth were then treated with chemo mechanical and traditional caries removal approach. Teeth were scanned with the same voxel sizes using micro-computed tomography images to figure out the difference dentin mineral density and to calculate the removed dentinal volume after chemo-mechanical or traditional method application.

Results: The results showed increase of cavity volume in the chemo-mechanical and traditional method groups. No significant difference was found for cavity volume and dentinal carious volume in both groups ( $p>0.05)$. The mineral density values of demineralized dentin were between $0.52-0.66 \mathrm{~g} / \mathrm{cm}^{3}$ before caries removal, and $1.39-1.59 \mathrm{~g} / \mathrm{cm}^{3}$ after removing caries. These values were found to be within the range of healthy dentin mineral density values again without any significant difference between groups ( $\mathrm{p}>0.05$ ).

Conclusion: In conclusion, chemo-mechanical methods can be used effectively for removing the caries in the primary molar teeth similar to conservative treatments.
\end{abstract}

Key words: caries removing techniques; micro-CT; Papacarie; residual caries

\section{Introduction}

Minimally invasive dentistry is mainly focused on the early diagnosis of caries lesions, reduction of caries progression by improvements in materials and techniques, and prevention of these lesions or restorative treatment of dental tissues with minimal damage. ${ }^{1-3}$ The concept of minimally invasive dentistry has become increasingly popular due to the presence of new restorative materials, improved understanding of the carious process, and enhanced abilities to utilize the remineralization potentials of enamel and dentin. 4 Black's "Extension for Protection" principle, which is based on determining the cavity dimensions using the traditional method, is replaced with the "Prevention of Extension" principle in minimally invasive dentistry. ${ }^{5-7}$

Minimally invasive dentistry is a selective caries removal method that focuses on preserving dental tissue with the potential for remineralization, as well as the removal of infected and denatured dentin. ${ }^{8}$ Clinical differentiation of infected and affected 
dentin is difficult and may result in under- or over-excavation. ${ }^{9}$ In the traditional method, carious dentin is removed using a slowspeed round diamond, tungsten carbide, or carbon-steel bur, and clinicians determine the dimension of the cavity by the hardness and color of the dentin. ${ }^{9,10}$ However, a more objective caries detection dye ( $1 \%$ acid red solution in propylene glycol; Caries Detector, Kuraray, Osaka, Japan) is recommended to determine the endpoint of decay removal. ${ }^{11}$

Many techniques and materials have been introduced for cavity preparation and caries removal as minimally invasive alternatives to the traditional method, including the hall technique, non-restorative caries treatment, air-abrasion, enzymes, airpolishing, ultrasonic instrumentation, sono-abrasion, laser techniques, fluorescence-aided caries excavation, and chemo mechanical caries removal. ${ }^{12-15}$ All these approaches are expected to result in high sensitivity, minimal pain, and the preservation of intact, uninfected dentin. ${ }^{12}$

Chemo mechanical caries removal has become an alternative to the traditional method especially for pediatric patients, as well as patients with anxiety or disabilities. ${ }^{16-18}$ In this minimally invasive dentistry technique, infected dentin is chemically softened using a chemo-mechanical agent then gently removed with an excavator. ${ }^{19-22}$ Chemo-mechanical caries removal agents include sodium hypochlorite-based agents (e.g., GK-10, GK-101E [Caridex ${ }^{\mathrm{TM}}$ ], and Carisolv $^{\mathrm{TM}}$ ) and enzyme-based agents (e.g., Papacarie ${ }^{\mathrm{TM}}$, Carie Care, and Biosolv). ${ }^{23}$

In 2003, Papacarie was introduced in Brazil as a chemomechanical caries removal agent containing papain, chloramine, and toluidine blue. Papacarie contributes to degradation by using papain to breaking collagen bonds, which are protected in healthy tissue by alpha-1 anti-trypsin, an enzyme absents from infected tissue. Therefore, polypeptide chains are cleaved, and collagen fibril cross-links are hydrolyzed. ${ }^{21}$ Papacarie Duo has become a widely used agent for conservative caries removal. In studies of its clinical efficacy, dentin remaining after caries removal with papain gel had similar microbiological characteristics to dentin remaining after use of the traditional method. ${ }^{21}$ Micro-computed tomography (CT) is a research technique that allows hard tissue volumetric measurements and mineral density evaluation without damaging samples. This technique is gaining increasing popularity in dental research. ${ }^{10,24-26}$ Studies have been published comparing caries removal method efficacies by means of micro-CT. ${ }^{27,28}$ However, to the best of our knowledge, Papacarie Duo has not been compared with conventional methods in terms of caries removal efficacy. Here, we evaluated dentin mineral density before and after caries removal with the traditional caries removal technique and Papacarie Duo method, with or without the use of caries detection dye. Our null hypothesis was that the Papacarie Duo method with minimally invasive treatment would achieve an effect similar to that of conservative pediatric dentistry treatment.

\section{Materials and Methods}

Our study protocol was approved by the Ankara University Faculty of Dentistry Clinical Research Ethics Committee (36290660/74 number 19.01.2016). Pediatric patients and their parents were informed of the research and provided written informed consent.

\section{Determination of sample size}

To determine an appropriate sample volume, a power analysis was performed before the study began. The analysis indicated that, when a difference of at least $0.2 \mathrm{~g} / \mathrm{cm}^{3}$ was present in mean mineral density between groups, at least 12 teeth per group were needed to detect this difference with $90 \%$ power at a type I error rate of $5 \%$.

\section{Groups}

The two main study groups underwent treatment with the Papacarie Duo or traditional method. These two groups were subdivided based on caries detection dye application.

\section{Interventions}

In accordance with the caries classification by Mount ${ }^{29}$ concerning caries distribution area and progression of the lesion, as well as periapical radiographic examination results, teeth with mediumsize and approximal caries were included in our study. The teeth were stored in saline solution for a maximum of 3 months after the surface residues had been cleaned. Teeth with no dentin caries involvement and those with carious lesions $<1 \mathrm{~mm}$ from the pulp chamber were excluded. In the Papacarie Duo group (group 1a), only Papacarie Duo gel was used for caries removal, in accordance with the manufacturer's recommendations. The cavity was filled with gel and incubated for 40-60 s. The softened tissue was then removed with an excavator. The application was repeated until a clear gel was obtained and the dentin decay in the teeth was evaluated by the operator using tactile criteria. The remaining gel was removed with moist cotton pellets and the cavity was washed with an air-water spray.

In the Papacarie Duo plus caries detection dye group (group 1b), caries detection was performed using a caries detector (Kuraray, Osaka, Japan), in accordance with the manufacturer's recommendations. The cavity surface was dried with air-water spray, and a $1 \%$ acid red caries dye was applied to the cavity using a disposable sponge tip applicator. The caries detection dye was incubated in the cavity for $10 \mathrm{~s}$, then washed with water and dried with air-water spray. Papacarie Duo gel was used for caries removal as in group 1a. Only dark pink-stained infected dentin was removed, and light pink-stained affected dentin was preserved. The remaining gel was removed with moist cotton pellets and the cavity was washed with air-water spray. In the traditional method group (group 2a), caries removal was performed using a low-speed rotary handpiece (KaVo Intramatic $20 \mathrm{ES}$; Kavo Dental GmbH, Biberach, Germany) and a \#016 steel round drill (Meisinger, Hager \& Meisinger, Düsseldorf, Germany). For each tooth, a new drill was used. Complete caries excavation was confirmed by tactile and visual methods.

In the traditional method plus caries detection dye group (group 2b), caries detection was performed using a caries detector (Kuraray Co., Ltd., Tokyo, Japan), in accordance with the manufacturer's recommendations. Only dark pink-stained infected dentin was removed. Light pink-stained affected dentin was preserved. The traditional method was used for caries removal as in group $2 \mathrm{a}$.

\section{Micro-CT evaluation}

A Bruker SkyScan 1174 high-resolution, desktop micro-CT system (SkyScan, Kontich, Belgium) was used to scan the specimens. The scanning conditions were: $50 \mathrm{kVp}, 100 \mathrm{~mA}$ beam current, $0.5 \mathrm{~mm}$ $\mathrm{Al} / \mathrm{Cu}$ filter, $15 \mu \mathrm{m}$ pixel size, and 0.5 step rotation. To minimize ring artifacts, air calibration of the detector was performed before each scan. Each sample was rotated $180^{\circ}$ during an integration period of $5 \mathrm{~min}$. The mean scanning duration was approximately 1 h. Other settings included beam-hardening correction and input of optimal contrast limits, both in accordance with the manufacturer's recommendations, based on prior scans and reconstructions of the teeth. Each tooth was scanned two times before and after the application of procedures to ensure standardization. 


\section{Micro-CT image analysis}

NRecon (ver. 1.6.9; SkyScan) and CTAn (ver. 1.14.1.2; SkyScan) were used for visualization and quantitative measurement of the samples, based on a modified version of the algorithm described by Feldkamp et al. 30 to obtain two-dimensional axial images (1000 $\times$ 1000 pixels). The reconstruction parameters were zero ring artifact correction, zero smoothing, and $40 \%$ beam artifact correction. Contrast limits were applied in accordance with the manufacturer's recommendations. Using NRecon software (Skyscan), images obtained by the scanner were reconstructed to show two-dimensional slices of the roots. In total, approximately 900 cross-sectional images were reconstructed from each whole volume. CTAn (Skyscan) software was then used for three-dimensional volumetric visualization and analysis. All reconstructions were performed with a 21.3-inch flat-panel color-active matrix TFT medical display (NEC MultiSync MD215MG, Munich, Germany), which had a resolution of $2048-2560$ at $75 \mathrm{~Hz}$ and $0.17-\mathrm{mm}$ dot pitch operated at 11.9 bits.

Skyscan CTAn software enables integrated calibration of datasets into two density scales: Hounsfield unit and mineral density. Therefore, two mineral concentration conical mineral density phantoms (rods) of 0.25 and 0.75 gHAp $\mathrm{cm}^{3}$ were used for calibration scans and measurements. To aid in calibration, the samples were scanned concurrently with mineral density phantom rods in identical tubes, which allowed density values to be independently calibrated for each scan. After scanning, grayscale values were converted to mineral density values using a linear calibration curve based on grayscale values obtained from the two mineral concentration conical phantoms. All scan parameters (e.g., pixel size, rotation step, frame averaging, voltage, and filter) were identical for all scans.

Tooth samples were scanned before and after caries removal at identical values with HAP phantoms. The teeth were scanned at least 2-3 $\mathrm{mm}$ apical to the cementoenamel junction. To distinguish enamel from other structures, a suitable threshold was required. Therefore, global thresholding was applied using semi-automated procedures in CTAn software. For analysis of dentinal mineral density in three-dimensional volumes, the original grayscale images were processed with a Gaussian low-pass filter for noise reduction, then subjected to an automatic segmentation threshold. The thresholding (binarization) process involved processing the range of gray levels to obtain an image of black/white pixels only. For each slice, a separate region of interest was selected to analyze the mineral density (Figure 1).

In order to determine the caries removal efficiency, the values defined in the previous studies in which the mineral density of the intact dentine with micro-CT and dentin before and after the caries removal were evaluated was taken as basis, and the solid dentin mineral density threshold value was accepted as $1.11 \mathrm{~g} / \mathrm{cm}^{3}{ }^{10,25}$

Calculations of mineral loss difference $\left(\Delta \mathrm{Z} ; \mathrm{gHAp} \mathrm{cm}^{3}\right)$ in each specimen were made by calculating the mineral density values from the entire demineralized area as the mean surface loss and mean loss of inner demineralized area (Figure 2). Mineral loss was calculated by subtracting demineralized mineral density values from baseline phantom rod mineral density values to correct alignment inaccuracies from the specimen. Densities were compared among all regions of interest. Tooth tissue with mineral density less than or equal to the selected threshold was considered as carious tissue. ${ }^{30}$

Micro-CT data obtained from before and after caries removal using Papacarie Duo and traditional methods images were reconstructed (NRecon version 1.6.9.4, Skyscan, Kontich, Belgium). During reconstruction, the light curing rate, ring artifact reduction, smoothing, frame avering were individually adjusted to the optimum value for each sample. 8-bit gray value images were obtained as a result of the reconstruction of the raw data. 8-bit gray images reconstructed using NRecon were transferred to CTAn (version 1.13.5.1) software. In the CTAn software, the images of each sample before and after the caries removal process are opened separately.
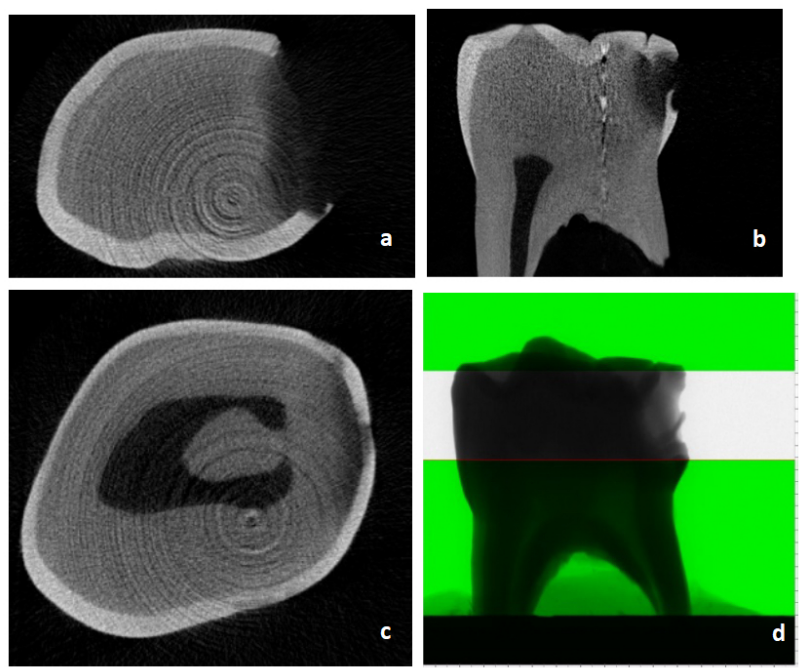

Figure 1. Imaging of the sample by micro-CT. a; transaxial section was taken proximal to the crown. $\mathbf{b}$; A sagittal section of the tooth. $\mathbf{c}$; A transaxial section from the pulp chamber level of the crown. $\mathbf{d}$; Determination of the upper and lower limits of the cavity area before the three-dimensional analysis.

By determining the cavity boundaries, the upper and lower boundaries were determined for analysis, and the Region of Interest (ROI) was selected by determining the cavity areas from the images in between. "Cavity Volume" was obtained from the selected ROI regions with three-dimensional images via CTAn software.

\section{Statistical analysis}

Statistical analyses were performed using SPSS Statistics for Windows, version 22.0 (IBM Corp., Armonk, NY, USA). The Kolmogorov-Smirnov test was used to analyze the normality of mineral density data. Levene's test was used to assess homogeneity of variances. Differences in mineral density values between groups and within groups were investigated by repeated analysis of variance. Statistical significance was regarded as $\mathrm{p}<0.05$.

\section{Results}

The before and after mineral densities in all groups are shown in Table 1 . The mean density increases in groups $2 \mathrm{a}$ and $2 \mathrm{~b}$ were 0.93 $\mathrm{g} / \mathrm{cm}^{3}$ and $0.99 \mathrm{~g} / \mathrm{cm}^{3}(\mathrm{p}<0.05)$. However, no significant difference was observed in mean density increases between groups $2 \mathrm{a}$ and $2 \mathrm{~b}$ ( $p>0.05$ ). Additionally, there were no significant differences in mineral concentrations of teeth between groups $1 \mathrm{a}$ and $2 \mathrm{a}$ or groups $1 b$ and $2 b$, before and after caries removal $(p>0.05)$.

After the caries detection dye was applied to the cavities, caries removal procedures were carried out for each group. In Group 1a, when the cavity volumes were compared before and after removing, an average increase in volume of $2.61 \mathrm{~mm}^{3}$ was found, but no statistically significant increase was found ( $p>0.05$ ). In group $1 b$, when the cavity volumes were compared before and after removing, an average volume increase of $6.91 \mathrm{~mm}^{3}$ was found and this increase was found to be statistically significant $(p<0.05)$. When Group 1a and Group 1b were compared, no statistically significant difference was found ( $p>0.05$ ). When the cavity volumes were compared before and after removing in group $2 \mathrm{a}$, a statistically significant increase of an average of $6.16 \mathrm{~mm}^{3}$ was found $(\mathrm{p}<0.05)$. In group $2 \mathrm{~b}$, when the cavity volumes were compared before and after the caries was removed, a statistically significant increase of $8.67 \mathrm{~mm}^{3}$ was found ( $\mathrm{p}<0.05$ ). When Group $2 \mathrm{a}$ and Group $2 \mathrm{~b}$ were compared, no statistically significant difference was found ( $p>0.05$ ). The cavity volumes of the teeth in Group 1a and Group 


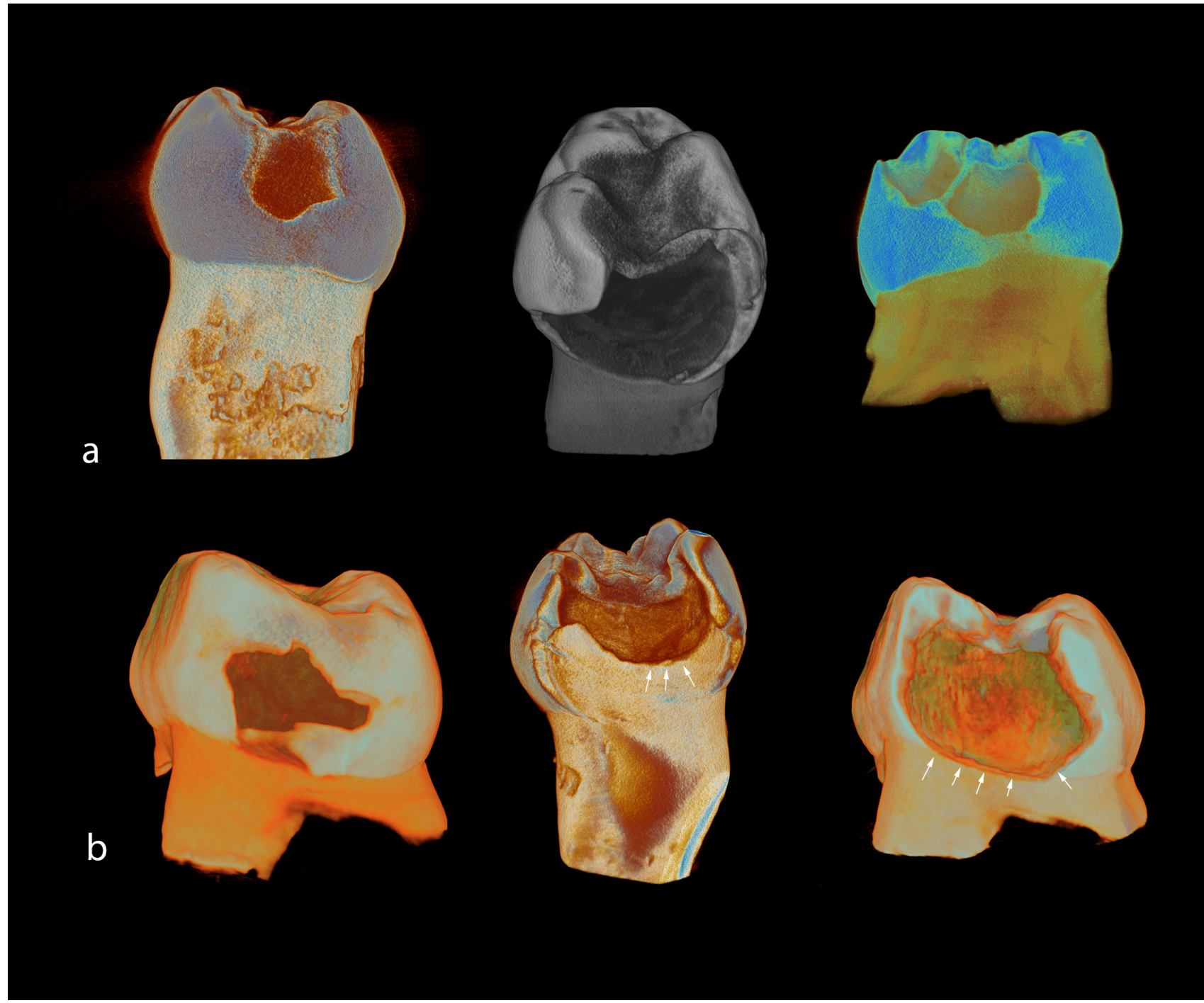

Figure 2. Volume renderings of micro-CT sections of baseline and treated teeth calibrated for dentin mineral density values $\left(\mathrm{g} / \mathrm{cm}^{3}\right)$. a; $3 \mathrm{D}$ volume rendering of baseline tooth; b; 3D volume rendering of treated tooth Arrows point to locations of carious dentin at the threshold for removal.

2a before and after the application were comparatively evaluated in Table 2. When the cavity volumes of the teeth in Group 1a and Group 2a were compared before and after removing, no statistically significant difference was found ( $p>0.05$ ). The cavity volumes of the teeth in Group $1 \mathrm{~b}$ and Group $2 \mathrm{~b}$ before and after the application were evaluated comparatively in Table 2 . When the cavity volumes of the teeth in Group $1 \mathrm{~b}$ and Group $2 \mathrm{~b}$ were compared before and after removing, no statistically significant difference was found ( $p>$ 0.05).

\section{Discussion}

In this study, it was aimed to investigate an effective caries removal method in which healthy dental tissue can be preserved by chemo-mechanical caries removal method and in which local anesthesia and rotary instruments which lead to heat, pressure and vibration were not used to pediatric patient. The caries removal efficiency of traditional rotary instrument and chemo-mechanical caries removal technique was evaluated with micro-CT and it was investigated whether it could be an alternative method for pediatric dentistry. Laser and chemo-mechanical caries removal methods both protect healthy tooth tissue, are more acceptable to patients, and require less use of local anesthesia, compared with the traditional method. ${ }^{31,32}$ Other alternative methods include air abrasion, air polishing, sono-abrasion, and ultrasonic approaches, although these methods carry some disadvantages that discourage their use in clinical practice. ${ }^{9,33}$ According to the results of our study, it was observed that when Papacarie Duo was applied to remove the caries in the primary molar teeth, caries can be removed effectively, and conservative treatments can be made without removing unnecessary tissue.

Using cone-beam CT to compare changes in volume and time elapsed for each technique, before and after caries removal, Thomas et al. ${ }^{34}$ showed that the chemo-mechanical caries removal method was the most effective minimally invasive technique, compared with other available techniques. They also noted that this method supported treatment acceptance by pediatric patients. Furthermore, Flückiger et al. ${ }^{33} 2005$ evaluated the caries removal efficiencies of atraumatic restorative treatment (ART) and chemo-mechanical caries removal methods (Carisolv ${ }^{\mathrm{TM}}$ ) on deciduous teeth. They showed that the chemo-mechanical method preserved tissue and effectively removed caries. In a comparison of three caries removal methods (ART, Carisolv ${ }^{\mathrm{TM}}$, and Papacarie), Boob et al. ${ }^{35}$ demonstrated stated that the most effective caries removal was achieved with chemo-mechanical methods. Coahuila et al. ${ }^{36}$ evaluated the behavior of Papacarie during and after the treatment of dental caries in pediatric patients. The good tolerance of Papacarie indicated that it constituted a minimally invasive and atraumatic method.

To evaluate mineral density in dental hard tissues, chemical 
Table 1. Mean mineral densities of dentin before and after caries removal using Papacarie Duo and traditional methods (mean \pm standard deviation)

\begin{tabular}{|c|c|c|c|c|}
\hline \multirow[b]{2}{*}{ Mineral density $\left(\mathrm{g} / \mathrm{cm}^{3}\right)$} & \multicolumn{2}{|c|}{ Papacarie Duo } & \multicolumn{2}{|c|}{ Traditional method } \\
\hline & Papacarie Duo & $\begin{array}{c}\text { Papacarie Duo plus caries } \\
\text { detection dye }\end{array}$ & Traditional method & $\begin{array}{l}\text { Traditional method plus } \\
\text { caries detection dye }\end{array}$ \\
\hline Before caries removal & $0.60( \pm 0.24)^{\mathrm{Aa}}$ & $0.52( \pm 0.58)^{\mathrm{Aa}}$ & $0.66( \pm 0.33)^{\mathrm{Aa}}$ & $0.54( \pm 0.33)^{\mathrm{Aa}}$ \\
\hline After caries removal & $1.39( \pm 0.31)^{\mathrm{Ab}}$ & $1.47( \pm 0.25)^{\mathrm{Ab}}$ & $1.59( \pm 0.18)^{\mathrm{Ab}}$ & $1.53( \pm 0.34)^{\mathrm{Ab}}$ \\
\hline
\end{tabular}

Different capital letters in each line indicate a statistically significant difference $(\mathrm{p}<0.05)$. Different lowercase letters in each column indicate a statistically significant difference $(\mathrm{p}<0.05)$.

Table 2. Cavity Volume $\left(\mathrm{mm}^{3}\right)$ of dentin before and after caries removal using Papacarie Duo and traditional methods (mean \pm standard deviation)

\begin{tabular}{l|c|c|c}
\hline & \multicolumn{2}{|c|}{ Papacarie Duo } \\
Mineral density $\left(\mathrm{g} / \mathrm{cm}^{3}\right)$ & Papacarie Duo & $\begin{array}{c}\text { Traditional method } \\
\text { Traditional method plus } \\
\text { detection dye }\end{array}$ & $\begin{array}{c}\text { Traditional method } \\
\text { caries detection dye }\end{array}$ \\
\hline Before caries removal & $19,92( \pm 12,09)^{\mathrm{Aa}}$ & $\begin{array}{c}10,06( \pm 5,69)^{\mathrm{Aa}} \\
16,97( \pm 11,48)^{\mathrm{Ab}}\end{array}$ & $\begin{array}{c}16,25( \pm 9,43)^{\mathrm{Aa}} \\
22,41( \pm 11,88)^{\mathrm{Ab}}\end{array}$ \\
After caries removal & $22,53( \pm 13,12)^{\mathrm{Aa}}$ & $\begin{array}{c}15,90( \pm 8,18)^{\mathrm{Aa}} \\
24,57( \pm 14,07)^{\mathrm{Ab}}\end{array}$ \\
\hline
\end{tabular}

Different capital letters in each line indicate a statistically significant difference $(\mathrm{p}<0.05)$. Different lowercase letters in each column indicate a statistically significant difference $(\mathrm{p}<0.05)$.

and micro-radiographic techniques are needed. ${ }^{37}$ Transverse microradiography is considered the gold standard for the determination of mineral contents and caries lesion depth. However, these analysis techniques are destructive and slow when identifying the original surface of a dentin lesion. Moreover, air drying of a dentin lesion can result in characteristic surface shrinkage. In recent years, the micro-CT system has provided a reliable method for the measurement of bone and tooth mineral densities. ${ }^{37}$ Miyajima et al. ${ }^{38}$ reported the potential for micro-CT to replace transverse microradiography within in vitro studies of tooth caries because micro-CT can be used with intact samples, provides three-dimensional images, and shows structural and composition changes in real-time.

In previous studies, the mean mineral densities of intact and demineralized primary teeth dentin were $1.29 \mathrm{~g} / \mathrm{cm}^{3}$ and 0.55 $\mathrm{g} / \mathrm{cm}^{3}$, respectively. ${ }^{2}$ In our study, the mineral densities of demineralized dentin were between $0.52-0.66 \mathrm{~g} / \mathrm{cm}^{3}$ before caries removal, similar to the values in previous studies. Our results indicate that both Papacarie and traditional caries removal methods are effective. Neves et al. ${ }^{10}$ evaluated nine carries removal methods with micro-CT. Their results were similar to ours in that the residual dentin mineral density was higher after traditional caries removal than after chemo-mechanical caries removal. Furthermore, AlHumaid et al. ${ }^{27}$ compared the removed tissue volume and mineral density of remaining dentin by using X-ray microtomography after caries removal with Papacarie and Carisolv ${ }^{\mathrm{TM}}$. Compared with Carisolv $^{\mathrm{TM}}$, the use of Papacarie caused less tissue removal and produced healthier dentin. The mineral density values of dentin, following caries removal with Papacarie, were in a similar range of values to those observed in our study $\left(1.45 \times 0.17 \mathrm{~g} / \mathrm{cm}^{3}\right)$. Djomehri et al. ${ }^{39}$ also investigated the Carisolv ${ }^{\mathrm{TM}}$ and Papacarie methods, and showed low mineral density after excavation.

Because micro-CT is a new method, there are no reference mineral density values available for caries assessment. Compared with the traditional method, a more irregular surface is obtained after chemo-mechanical caries removal. Hamama et al. ${ }^{40}$ reported that the remaining dentin layer was the main difference between $\mathrm{Pa}$ pacarie and rotary instruments. In this layer, the mineral density is lower than that of intact dentin, but higher than that of infected dentin. They defined this remaining dentin layer as affected dentin, which could be retained without compromising the success of the restoration. Clementino-Luedemann et al. ${ }^{41} 2006$ reported that minerals accumulated in this residual dentin layer after the chemomechanical removal of caries, which impeded further caries progression. Previous studies showed that despite caries removal, mineral densities could be lower after coverage with healthy dentin. 42 The comparison of mineral densities between the caries removal methods in our study showed that they were higher after use of the traditional method than after use of the Papacarie Duo method. This result is consistent with prior findings. The results showed that the chemo-mechanical agents showed minimal change in cavity volume when compared to traditional method. When the cavity volume was compared before and after the caries removal techniques with the caries detection dye were applied, it was found that the cavity volume increased the most after the caries detection dye was applied in the traditional method.

Similar to the results of our study, according to the results of the study in which Neves et al. ${ }^{26}$ evaluated the effectiveness of nine different caries removal methods with Micro-CT, it was observed that more tissue was removed in the group in which the caries detection dye was used, and the group in which only the traditional method was applied was more conservative compared to the group using caries detection dye. According to the results of the same study, it was determined that it is the most minimally invasive technique in chemo-mechanical caries removal techniques as less intact tooth tissue is removed. In our study, it was observed that only the Papacarie Duo group had an increase in the cavity volume at least compared to the other groups and effectively removed caries. In parallel with these results, few problems experienced as it took very long time that finding proper manufacturer and then shipment of the material. It was also reported that Genotoxicity was not tested for Papacarie Duo. In future studies, it is necessary to compare samples with high number of samples containing cavities of various sizes.

\section{Conclusion}

The chemo mechanical caries removal has strong potential for use in minimally invasive dentistry, particularly in the removal of caries from primary teeth. Infected dentin can be effectively removed, and more conservative treatments can be performed without the risk of pulp perforation.

\section{Acknowledgements}

The work was supported by the Ankara University, Scientific and Research Fund with project number "16H0234001". 


\section{Author Contributions}

MTC and FTO conceived the ideas; MTC collected the data and performed the study MO, KO analyzed the data; and MTV and KO. led the writing.

\section{Conflict of Interest}

The authors declare no conflicts of interest associated with this manuscript.

\section{Authors' ORCID(s)}

M.T.C. $\quad 0000-0002-2734-9873$

F.T.O. $\quad 0000-0002-8731-5907$

M.O. $\quad 0000-0001-6832-6208$

K.O. $\quad 0000-0001-6768-0176$

\section{References}

1. Featherstone JD. The science and practice of caries prevention. J Am Dent Assoc. 2000;131(7):887-99. doi:10.14219/jada.archive.2000.0307.

2. Featherstone JD, Doméjean S. Minimal intervention dentistry: part 1. From 'compulsive' restorative dentistry to rational therapeutic strategies. Br Dent J. 2012;213(9):441-5. doi:10.1038/sj.bdj.2012.1007.

3. Zero DT, Zandona AF, Vail MM, Spolnik KJ. Dental caries and pulpal disease. Dent Clin North Am. 2011;55(1):29-46. doi:10.1016/j.cden.2010.08.010.

4. Ericson D, Kidd E, McComb D, Mjör I, Noack MJ. Minimally Invasive Dentistry-concepts and techniques in cariology. Oral Health Prev Dent. 2003;1(1):59-72.

5. Burke FJ. From extension for prevention to prevention of extension: (minimal intervention dentistry). Dent Update. 2003;30(9):492-8, 500, 502. doi:10.12968/denu.2003.30.9.492.

6. Hamama HH, Yiu CK, Burrow MF, King NM. Chemical, morphological and microhardness changes of dentine after chemomechanical caries removal. Aust Dent J. 2013;58(3):283-92. doi:10.1111/adj.12093.

7. Peters MC, McLean ME. Minimally invasive operative care. I. Minimal intervention and concepts for minimally invasive cavity preparations. J Adhes Dent. 2001;3(1):7-16.

8. Mount GJ, Hume WR. A revised classification of carious lesions by site and size. Quintessence Int. 1997;28(5):301-3.

9. Banerjee A, Watson TF, Kidd EA. Dentine caries excavation: a review of current clinical techniques. Br Dent J. 2000;188(9):47682. doi:10.1038/sj.bdj.4800515.

10. Neves Ade A, Coutinho E, Vivan Cardoso M, Jaecques SV, Van Meerbeek B. Micro-CT based quantitative evaluation of caries excavation. Dent Mater. 2010;26(6):579-88. doi:10.1016/j.dental.2010.01.012.

11. Kidd EA, Joyston-Bechal S, Smith MM, Allan R, Howe L, Smith $\mathrm{SR}$. The use of a caries detector dye in cavity preparation. $\mathrm{Br}$ Dent J. 1989;167(4):132-4. doi:10.1038/sj.bdj.4806939.

12. El-Tekeya M, El-Habashy L, Mokhles N, El-Kimary E. Effectiveness of 2 chemomechanical caries removal methods on residual bacteria in dentin of primary teeth. Pediatr Dent. 2012;34(4):325-30.

13. Innes NP, Stirrups DR, Evans DJ, Hall N, Leggate M. A novel technique using preformed metal crowns for managing carious primary molars in general practice - a retrospective analysis. Br Dent J. 2006;200(8):451-4; discussion 444. doi:10.1038/sj.bdj.4813466.
14. Kidd E. Should deciduous teeth be restored? Reflections of a cariologist. Dent Update. 2012;39(3):159-62, 165-6. doi:10.12968/denu.2012.39.3.159.

15. Lennon AM. Fluorescence-aided caries excavation (FACE) compared to conventional method. Oper Dent. 2003;28(4):341-5.

16. Azrak B, Callaway A, Grundheber A, Stender E, Willershausen B. Comparison of the efficacy of chemomechanical caries removal (Carisolv) with that of conventional excavation in reducing the cariogenic flora. Int J Paediatr Dent. 2004;14(3):182-91. doi:10.1111/j.1365-263X.2004.00535.x.

17. Beeley JA, Yip HK, Stevenson AG. Chemochemical caries removal: a review of the techniques and latest developments. $\mathrm{Br}$ Dent J. 2000;188(8):427-30. doi:10.1038/sj.bdj.4800501.

18. Zhang X, Tu R, Yin W, Zhou X, Li X, Hu D. Micro-computerized tomography assessment of fluorescence aided caries excavation (FACE) technology: comparison with three other caries removal techniques. Aust Dent J. 2013;58(4):461-7. doi:10.1111/adj.12106.

19. Bussadori SK, Castro LC, Galvão AC. Papain gel: a new chemo-mechanical caries removal agent. J Clin Pediatr Dent. 2005;30(2):115-9. doi:10.17796/jcpd.30.2.xq641W720u101048.

20. Bussadori SK, Guedes CC, Bachiega JC, Santis TO, Motta LJ. Clinical and radiographic study of chemical-mechanical removal of caries using Papacárie: 24-month follow up. J Clin Pediatr Dent. 2011;35(3):251-4. doi:10.17796/jcpd.35.3.75803m02524625h5.

21. Bussadori S, Martins M, Fernandes K, Guedes C, Motta L, Reda $\mathrm{S}$, et al. Evaluation of in vitro biocompatibility of the new product for chemical-mechanical caries removal-Papacarie. Pesq Bras Odontoped Clin Integr Joao Pessoa. 2005;5(3):253-59.

22. Mhatre S, Sinha S, Bijle MNA, Thanawala EA. Chemomechanical caries removal system-A brief review. International Journal of Clinical Dental Science. 2011;2(2).

23. Maragakis GM, Hahn P, Hellwig E. Chemomechanical caries removal: a comprehensive review of the literature. Int Dent J. 2001;51(4):291-9. doi:10.1002/j.1875-595x.2001.tboo841.x.

24. Neboda C, Anthonappa RP, Engineer D, King NM, Abbott PV. Root canal morphology of hypomineralised first permanent molars using micro-CT. Eur Arch Paediatr Dent. 2020;21(2):229-240. doi:10.1007/s40368-019-00469-x.

25. Neves AB, Bergstrom TG, Fonseca-Gonçalves A, Dos Santos TMP, Lopes RT, de Almeida Neves A. Mineral density changes in bovine carious dentin after treatment with bioactive dental cements: a comparative micro-CT study. Clin Oral Investig. 2019;23(4):1865-1870. doi:10.1007/s00784-018-2644-2.

26. Neves Ade A, Coutinho E, De Munck J, Van Meerbeek B. Cariesremoval effectiveness and minimal-invasiveness potential of caries-excavation techniques: a micro-CT investigation. J Dent. 2011;39(2):154-62. doi:10.1016/j.jdent.2010.11.006.

27. AlHumaid J, Al-Harbi F, El Tantawi M, Elembaby A. Xray microtomography assessment of Carisolv and $\mathrm{Pa}-$ pacarie effect on dentin mineral density and amount of removed tissue. Acta Odontol Scand. 2018;76(4):236-240. doi:10.1080/00016357.2017.1406614.

28. Pires PM, Santos TPD, Fonseca-Gonçalves A, Pithon MM, Lopes RT, Neves AA. A dual energy micro-CT methodology for visualization and quantification of biofilm formation and dentin demineralization. Arch Oral Biol. 2018;85:10-15. doi:10.1016/j.archoralbio.2017.09.034.

29. Mount GJ. A new paradigm for operative dentistry. J Conserv Dent. 2008;11(1):3-10. doi:10.4103/0972-0707.43411.

30. Feldkamp LA, Goldstein SA, Parfitt AM, Jesion G, Kleerekoper $M$. The direct examination of three-dimensional bone architecture in vitro by computed tomography. J Bone Miner Res. 1989;4(1):3-11. doi:10.1002/jbmr.5650040103.

31. Ganesh M, Parikh D. Chemomechanical caries removal (CMCR) agents: Review and clinical application in primary teeth. Journal of Dentistry and oral hygiene. 2011;3(3):34-45.

32. Martens LC. Laser physics and a review of laser applications in 
dentistry for children. Eur Arch Paediatr Dent. 2011;12(2):61-7. doi:10.1007/bf03262781.

33. Flückiger L, Waltimo T, Stich $\mathrm{H}$, Lussi A. Comparison of chemomechanical caries removal using Carisolv or conventional hand excavation in deciduous teeth in vitro. J Dent. 2005;33(2):8790. doi:10.1016/j.jdent.2004.07.007.

34. Thomas AR, Nagraj SK, Mani R, Haribabu R. Comparative evaluation of the efficiency of caries removal using various minimally invasive techniques with conventional rotary instruments using cone beam computed tomography: An in vitro study. Journal of International Oral Health. 2020;12(3):253.

35. Boob AR, Manjula M, Reddy ER, Srilaxmi N, Rani T. Evaluation of the Efficiency and Effectiveness of Three Minimally Invasive Methods of Caries Removal: An in vitro Study. Int J Clin Pediatr Dent. 2014;7(1):11-8. doi:10.5005/jp-journals-10005-1226.

36. Coahuila FdGA, Martínez DLN, Carrillo EL, Rayón LS. Acceptance of chemical-mechanical caries removal by four-to six-year-old patients seen in 2018. Archivos de Investigación Materno Infantil. 2019;10(1):22-29.

37. Swain MV, Xue J. State of the art of Micro-CT applications in dental research. Int J Oral Sci. 2009;1(4):177-88. doi:10.4248/ijos09031.

38. Miyajima $\mathrm{H}$, Ishimoto $\mathrm{T}, \mathrm{Ma} \mathrm{S}$, Chen $\mathrm{J}$, Nakano $\mathrm{T}$, Imazato S. In vitro assessment of a calcium-fluoroaluminosilicate glass-based desensitizer for the prevention of root surface demineralization. Dent Mater J. 2016;35(3):399-407. doi:10.4012/dmj.2015-273.

39. Djomehri SI, Candell S, Case T, Browning A, Marshall GW, Yun $\mathrm{W}$, et al. Mineral density volume gradients in normal and diseased human tissues. PLoS One. 2015;10(4):e0121611. doi:10.1371/journal.pone.0121611.

40. Hamama HH, Yiu CK, Burrow MF. Caries management: A journey between Black's principals and minimally invasive concepts. International Journal of Dentistry and Oral Science (IJDOS). 2015;2(8):120-125.

41. Clementino-Luedemann TN, Kunzelmann KH. Mineral concentration of natural human teeth by a commercial micro-CT. Dent Mater J. 2006;25(1):113-9. doi:10.4012/dmj.25.113.

42. Hahn SK, Kim JW, Lee SH, Kim CC, Hahn SH, Jang KT. Microcomputed tomographic assessment of chemomechanical caries removal. Caries Res. 2004;38(1):75-8. doi:10.1159/000073924. 